\title{
ESTUDIO PALINOLÓGICO DE LA FLORA URBANA DE LA CIUDAD DE CHIHUAHUA, MÉXICO
}

\author{
Alfredo Campos-Trujillo ${ }^{1,3}$, Anabel Aguirre-Prieto ${ }^{2}$, Giovanni Muñoz-Romero ${ }^{2}$, \\ Marco Antonio Rodríguez-Villa ${ }^{1}$ y Gustavo Quintana-Martínez ${ }^{2}$
}

${ }^{1}$ Centro de Investigación en Materiales Avanzados, Departamento de Energías Renovables y Protección del Medio Ambiente, Avenida Miguel de Cervantes

Número 120, 31190 Chihuahua, Chihuahua, México.

${ }^{2}$ Universidad Autónoma de Chihuahua, Facultad de Zootecnia y Ecología, km 1

Periférico Francisco R. Almada, 33820 Chihuahua, Chihuahua, México.

33Autor para la correspondencia: alfredo.campos@cimav.edu.mx

\section{RESUMEN}

El objetivo de este trabajo fue presentar el primer estudio palinológico de la ciudad de Chihuahua, México, resultando en el desarrollo de un documento gráfico del polen de las gimnospermas y angiospermas de la zona. Se incluyeron muestras tanto de plantas de ornato como silvestres, las cuales fueron colectadas en aceras, parques, áreas públicas y suburbios del área de estudio. El trabajo incluyó 91 especies: 15 árboles, 10 arbustos y 66 herbáceas correspondientes a 37 familias y 86 géneros. Los granos de polen fueron obtenidos directamente de los estambres y observados al microscopio electrónico de barrido sin acetolizar, hidratar o deshidratar. La mayoría de los granos de polen (60\%) estuvieron en el rango de tamaño medio. Los patrones de ornamentación más comunes fueron el equinado (23\%) y el reticulado (22\%). Un gran porcentaje mostró aberturas elongadas: tricolporadas (41\%) y tricolpadas (13\%), las cuales coinciden con el patrón de plegamiento con aberturas hundidas. Los granos de polen de la mayoría de las especies estudiadas presentan forma esferoidal en su estado hidratado (83\%), mientras que la más común entre los granos deshidratados es la prolata (51\%). Se observaron tres principales tipos polínicos; el primero corresponde a la familia Asteraceae, el segundo a Poaceae, mientras que el tercero incluye a 10 familias y 15 especies cuyos granos de polen presentan ornamentación reticulada, aberturas elongadas, forma prolata en su estado seco y aberturas hundidas. Se reconoce la necesidad de incluir a futuro más especies presentes en el área.

Palabras clave: fotomicrografías de polen, granos de polen, morfología, tipos polínicos. 


\begin{abstract}
The aim of this study was to provide the first palynological study of Chihuahua City, Mexico, resulting in the development of a graphic reference document about pollen of Gymnosperms and Angiosperms in this zone. Samples of both ornamental and wild plants were included, which were collected on sidewalks, parks, public areas, and suburbs of the studied area. The study included 91 taxa: 15 trees, 10 shrubs and 66 herbs from 37 families and 86 genera. Pollen grains were directly obtained from the stamens and observed with scanning electronic microscope without acetolizing, hydrating or dehydrating. Most of the studied pollen grains had a medium size (60\%). The most common ornamentation patterns were echinate (23\%) and reticulate (22\%). A large percentage of the pollen grains showed elongated apertures: tricolporate (41\%) and tricolpate (13\%), which coincides with the folding pattern of sunken apertures. Pollen grains of most of the studied species show spheroidal shape in hydrated state (83\%), while the most common shape among dehydrated grains is prolate (51\%). Three main pollen types were observed; the first type corresponds to the family Asteraceae and the second one to Poaceae. The third type included 10 families and 15 species whose pollen grains have reticulated ornamentation, elongated apertures, prolate shape in its dry state and sunken apertures. It is recognized that the inclusion of more species present in the area is needed.
\end{abstract}

Key words: morphology, pollen grains, pollen photomicrographs, pollen types.

\title{
INTRODUCCIÓN
}

La identificación de granos de polen se basa en la combinación de algunos caracteres tales como el tamaño, forma, estructura y ornamentación de la exina y sus aberturas. El conjunto de rasgos morfológicos es constante para cada especie, género o familia, lo que hace posible el reconocimiento del taxon de origen con una precisión adecuada. Sin embargo, la especie de la cual proviene el polen no siempre puede ser identificada, ya que en muchos casos dicha exactitud solo alcanza el nivel género o familia (Belmonte y Roure, 2002).

Los atlas palinológicos son útiles en la identificación de los granos de polen provenientes de muestras de aire usadas en aeropalinología, del colectado directamente de las plantas, así como del contenido en miel y en las abejas melíferas (Pino y Díez, 1993; Morais et al., 2011). Sin embargo, en ocasiones es difícil identificar o diferenciar ciertas formas de polen, lo cual puede resultar en la omisión de alguna especie indicadora importante (Adecanmbi, 2009). 
En México, algunos autores han estudiado la morfología del polen de diversas familias de plantas, especialmente en la región central del país (Arreguín-Sánchez et al., 1988 y 1990; Palacios-Chávez et al., 1996; Quiroz-García et al., 1997 y 2002; Goreti y Alfaro, 2003; Quiroz García y Arreguín Sánchez, 2004; Torres et al., 2010). Para la región Chihuahua se han realizado trabajos florísticos, como los de Vega (2008) y de Estrada-Castillón y Villarreal-Quintanilla (2010); sin embargo, ninguno de éstos se ha enfocado a la investigación del polen.

El objetivo general de este manuscrito fue contribuir al conocimiento palinológico de la ciudad de Chihuahua, México. Se presentan descripciones breves de los granos de polen, tomando en cuenta el tamaño, ornamentación, tipo de abertura, forma de los granos, así como el patrón de plegamiento (Apéndice).

Como objetivo particular se pretendió elaborar una guía de los granos de polen más representativos de la vegetación de la ciudad de Chihuahua, por lo que se incluyeron plantas tanto de ornato como silvestres. Tal guía también puede ser de utilidad en la identificación de polen proveniente de muestras ambientales, como los encontrados por Rodríguez-Villa (2009) en muestras de partículas suspendidas totales de la ciudad de Chihuahua (Fig. 1).

Este trabajo se realizó en el marco de un estudio más amplio sobre partículas atmosféricas, el cual incluyó la caracterización de las mismas por microscopía electrónica de barrido (MEB), por lo que la observación de los granos de polen se hizo únicamente con esta técnica.

\section{MATERIALES Y MÉTODOS}

La ciudad de Chihuahua se localiza en la región central del estado de Chi-

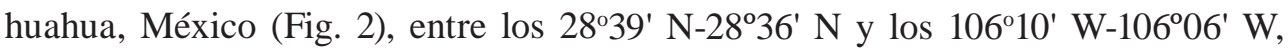
entre 1450 y 1320 m s.n.m. El clima es extremoso, seco a semiárido, con régimen
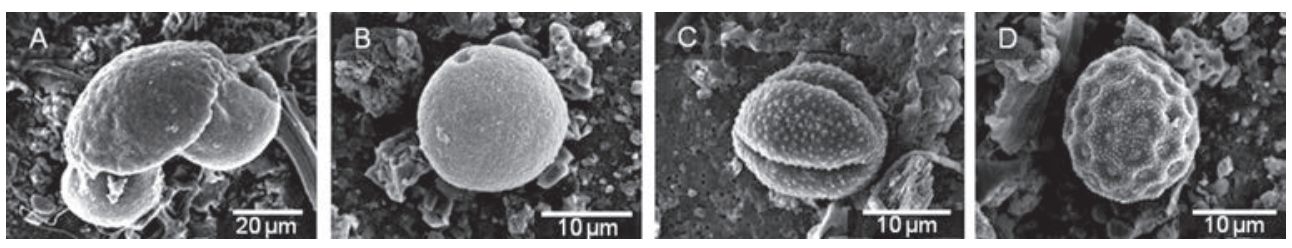

Fig. 1. Fotomicrografías de granos de polen encontrados en muestras de partículas atmosféricas en la ciudad de Chihuahua. A. Pinus spp.; B. Poaceae; C. Artemisia spp.; D. Chenopodiaceae. 
de lluvias en verano. La región recibe $320 \mathrm{~mm}$ de precipitación anual promedio. La temperatura media anual es de $18.6^{\circ} \mathrm{C}$ y la humedad relativa para un año promedio es de 47\% (Anónimo, 2000).

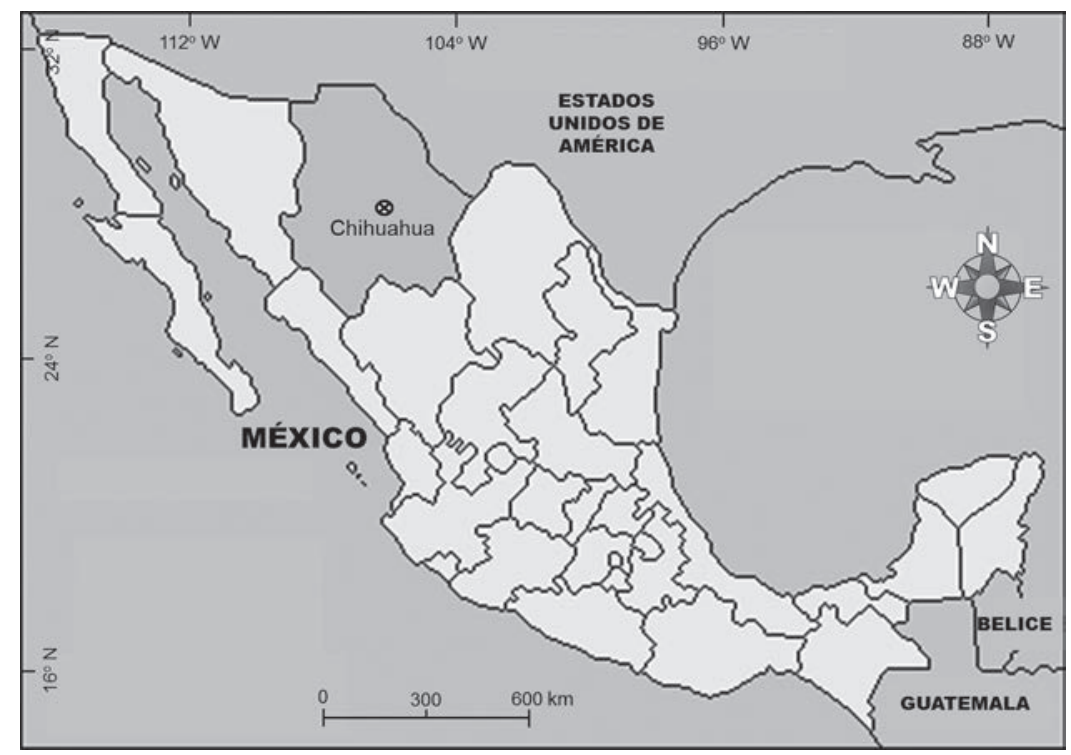

Fig. 2. Localización de la ciudad de Chihuahua, México.

Los tipos de vegetación típicos en los alrededores de la ciudad de Chihuahua son pastizales naturales e inducidos, combinados con áreas de matorral inerme. La flora consiste de plantas xerófilas, zacates, arbustos y árboles intercalados con algunas especies de agaves, yucas y cactáceas. En el área urbana pueden encontrarse plantas ornamentales, la mayoría de ellas introducidas.

La obtención de los especímenes fue realizada de marzo a octubre de 2007, y de febrero a marzo de 2008 por Aguirre y Muñoz (2009). Se recolectaron flores de las plantas que crecen en aceras, parques, áreas públicas, suburbios y alrededores de la ciudad de Chihuahua. De las 150 especies muestreadas, solo se pudo obtener polen de 91 de ellas, las cuales se presentan en este trabajo. Aunque no se tiene otro inventario más exhaustivo del área, se estima que se pueden encontrar en la ciudad alrededor de 200 géneros y unas 300 especies, por lo que este estudio representaría una tercera parte del total esperado. 
Para preparar los especímenes para su observación en el microscopio electrónico de barrido (MEB), los granos de polen fueron obtenidos de los estambres y montados directamente (sin acetolizar, hidratar o deshidratar) en portamuestras de aluminio con cinta conductiva de cobre y recubiertos con oro por la técnica de erosión catódica. Para examinar la morfología de los granos de polen se utilizó un MEB Jeol JSM-5800LV. Las fotomicrografías fueron obtenidas mediante electrones secundarios y un voltaje de aceleración de $10 \mathrm{kV}$.

La terminología utilizada en la descripción del polen se basó en Halbriter et al. (2007) y Punt et al. (2007), tomando en consideración la forma, tipo de abertura y patrón de ornamentación de la exina. Algunas descripciones se apoyaron en la base de datos palinológica Pal-Dat (Buchner y Weber, 2012) cuando estuvieron disponibles. Se consideraron cuatro tamaños: pequeño $(11-25 \mu \mathrm{m})$, mediano $(26-50 \mu \mathrm{m})$, grande $(51-100 \mu \mathrm{m})$ y muy grande $(>100 \mu \mathrm{m})$, de acuerdo con Erdtman (1952).

\section{RESULTADOS Y DISCUSIÓN}

En este estudio se incluyeron 91 especies (15 árboles, 10 arbustos, 66 herbáceas) pertenecientes a 1 familia de gimnospermas y 37 de angiospermas. Los caracteres generales de los granos de polen de las especies colectadas aparecen en forma abreviada en el Apéndice. Las fotomicrografías de los mismos se muestran en las láminas I a la IX.

Las familias con más especies en el conjunto de muestras fueron Asteraceae (18, 20\%), Poaceae (12, 13\%) y Fabaceae (10, 9\%). Estos resultados concuerdan con lo reportado en diferentes estudios florísticos realizados en el estado de Chihuahua (Lebge y Valerio, 1986; Estrada-Castillón, 1995; Spellenberg et al., 1996; Royo y Melgoza, 2001; Lebge et al., 2005; Vega, 2008 y Estrada-Castillón y VillarrealQuintanilla, 2010). Para México, Rzedowski (1998) y Villaseñor (2004) también reportan a las familias Asteraceae, Poaceae y Fabaceae como las de mayor número de géneros y especies.

Tamaño del polen. La mayoría de los granos de polen estudiados se encontraron dentro del rango de tamaño mediano (60\%). La segunda categoría más numerosa fue la pequeña (21\%), seguida de la grande con 15\%. Solo tres especies tuvieron tamaño muy grande: Acleisanthes longiflora (lámina VII, 13-14), Oenothera serrula (lámina VII, 23-24) y Oenothera gracilis (lámina VIII, 1-2). No se encontraron granos de polen de tamaño menor de $10 \mu \mathrm{m}$. 
Ornamentación. Los tipos de ornamentación observados fueron: baculado, clavado, equinado, microequinado, fosulado, lofado, microgemado, granulado, perforado, psilado, reticulado, microreticulado, retículo-cristado, rugulado, estriado y verrugado. Los más comunes fueron el equinado (21 especies, 23\%), en la familia Asteraceae (excepto en Gazania x splendens), y el reticulado, en 20 especies (22\%).

Tipos de abertura. Un gran porcentaje de los granos de polen fueron tricolporados (41\%). Doce especies (13\%) los presentaron tricolpados. La familia Poaceae comparte el característico tipo de abertura ulcerado-operculado-anulado. Esta familia representó $13 \%$ de las especies estudiadas (lámina II). 11\% de las especies tuvieron granos de polen inaperturados, mientras que el restante 22\% presentó diferentes tipos de aberturas (leptoma, monocolpado, pantoaperturado, pantocolpado, porado, pantoporado, triporado y sulcado).

Forma. Los granos de polen de la mayoría de las especies son esferoidales (83\%) en su estado hidratado. Otras formas encontradas fueron la prolata (9\%), irregular (4\%), oblata (3\%) y poligonal (1\%).

En cuanto a los granos de polen secos, la forma prolata fue la más común (51\%). Otras más fueron la esferoidal (28\%) y la irregular (10\%). Las oblata, triangular y en forma de bote sumaron el restante $11 \%$. La forma de los granos de polen en estado seco comúnmente es típica para una familia y/o género y por lo tanto es de relevancia sistemática (Hesse et al., 2009). Esta característica es importante en la identificación de muestras aerobiológicas, ya que en éstas es más probable encontrar granos de polen secos y plegados.

Plegamiento de los granos. La harmomegatia es el plegado característico de los granos de polen para ajustarse al decremento en el volumen celular debido a la perdida de agua. Durante la deshidratación, los granos de polen aperturados se vuelven cada vez más alargados, con los márgenes de la abertura aproximándose uno al otro de tal modo que ésta se sella efectivamente (Katifori et al., 2010). Alrededor de la mitad (51\%) de la especies presentaron este tipo de plegamiento, con la siguiente combinación de caracteres: aberturas elongadas (tricolporado, tricolpado y sulcado), forma prolata y aberturas hundidas en su estado seco.

Los granos de polen que carecen de aberturas elongadas responden a la desecación formando depresiones en la exina, cuyo número y localización varía grandemente. $49 \%$ de las especies mostró los siguientes patrones de plegamiento: irregular (22\%), en forma de copa (11\%), no plegado (8\%), aberturas hundidas no elongadas (5\%), área inaperturada hundida (3\%).

Tipos polínicos. Se observaron tres grandes grupos de granos de polen con caracteres morfológicos similares, los cuales abarcaron $47 \%$ de especies estudiadas. 
Dado que las familias Asteraceae y Poaceae fueron las más numerosas y que ambas son consideradas estenopolínicas, los tipos polínicos correspondientes a las mismas resultaron predominantes.

El primer grupo, conformado por 15 especies de la familia Asteraceae (excepto Gazania x splendens), presentó las siguientes características morfológicas: ornamentación equinada, aberturas tricolpadas o tricolporadas, prolados y con aberturas hundidas en su estado seco.

El segundo grupo, formado por las especies de la familia Poaceae, tiene tamaño mediano, ornamentación fosulada-granulada, aberturas ulceradas-operculadas-anuladas, forma esferoidal y plegamiento irregular. Solo las siguientes presentaron ligeras diferencias en su ornamentación, ya que su exina no es fosulada: Aristida adscensionis (microequinada), A. pansa (granulada) y Sorghum halepense (granulada).

El tercer grupo presentó ornamentación reticulada, aberturas tricolpadas, tricolporadas o triporadas, prolados en su estado seco y aberturas hundidas. Este tipo polínico incluyó a 10 familias y 16 especies: Adoxaceae: Sambucus nigra; Bignoniaceae: Tecoma stans; Brassicaceae: Capsella bursa-pastoris, Descurainia pinnata, Lepidium virginicum, Stanleya pinnata; Casuarinaceae: Casuarina equisetifolia; Fabaceae: Astragalus mollissimus, A. nuttallianus, Melilotus indicus, Parkinsonia aculeata; Fouquieriaceae: Fouquieria splendens; Oleaceae: Ligustrum japonicum; Papaveraceae: Argemone ochroleuca; Platanaceae: Platanus orientalis y Tamaricaceae: Tamarix gallica.
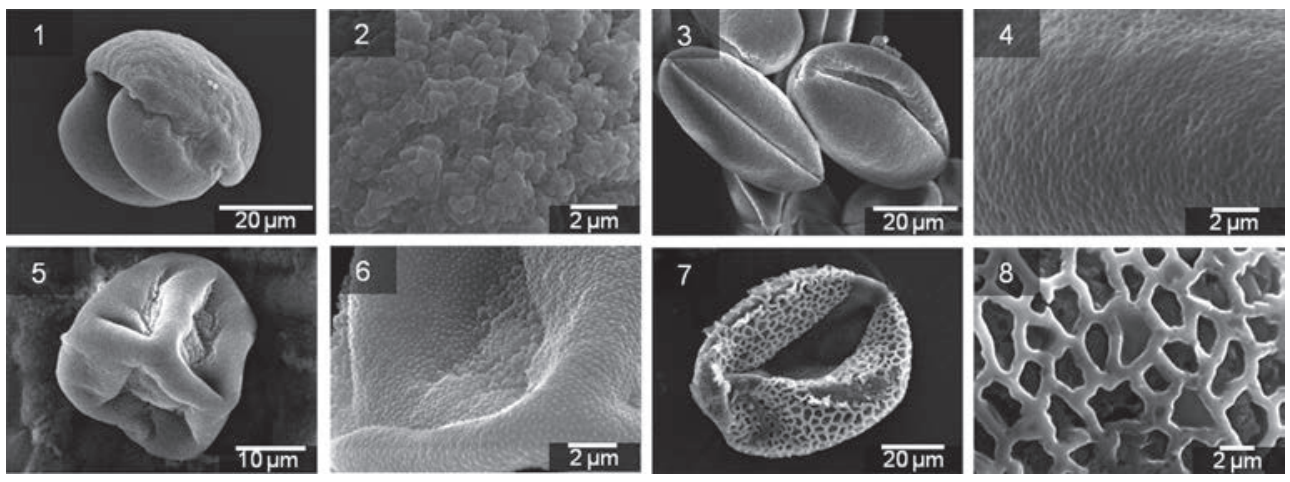

Lámina I. Pinus halepensis. 1. grano de polen (GP) vista ecuatorial; 2. superficie de la exina (SE). Yucca spp. 3. GP, vista ecuatorial oblicua; 4. SE. Cyperus esculentus. 5. GP; 6. SE, detalle de la abertura. Hermodactylus tuberosus. 7. GP, vista ecuatorial; 8. SE. 

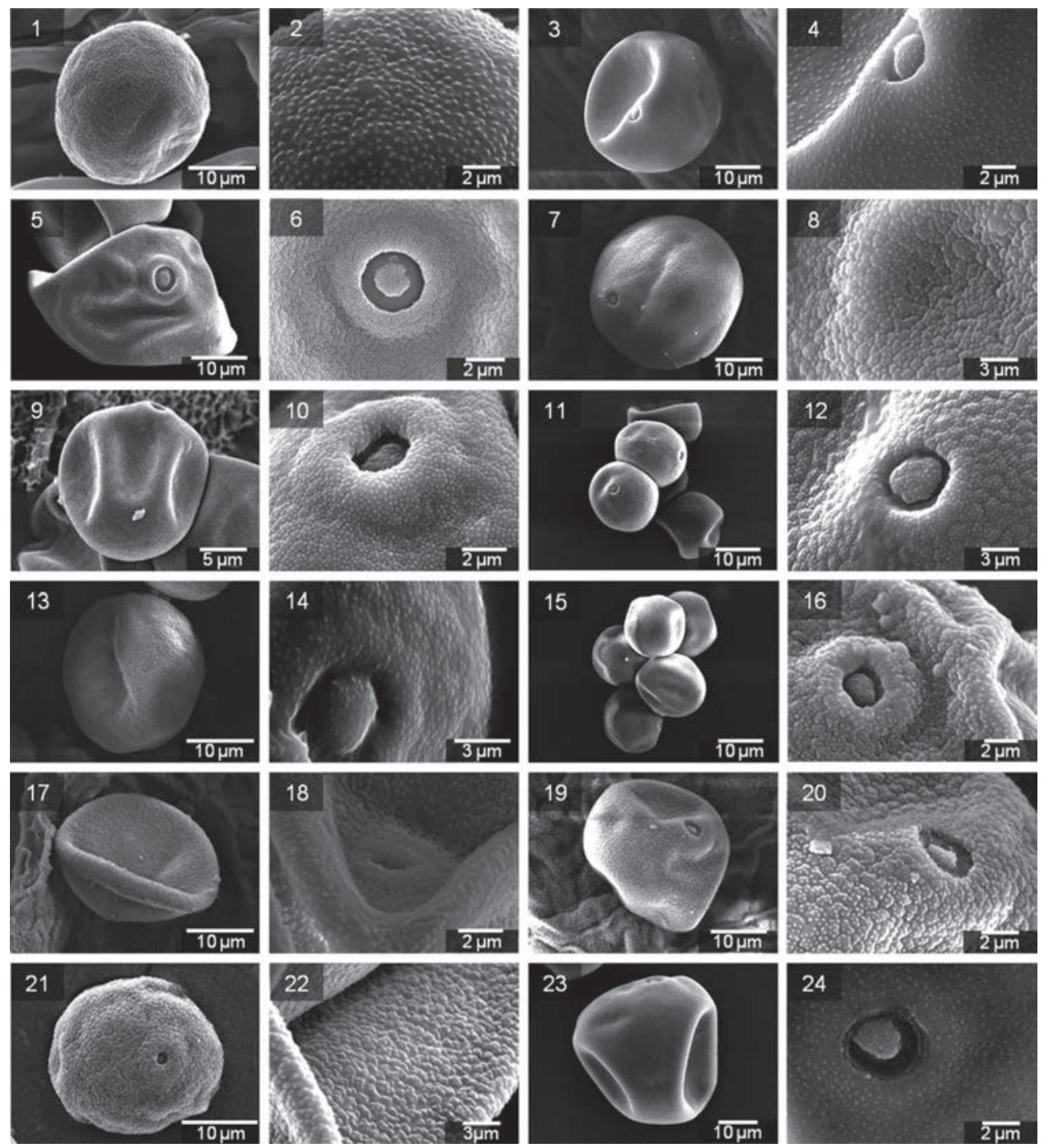

Lámina II. Aristida adscensionis. 1. grano de polen (GP), vista polar proximal; 2. SE. Aristida pansa. 3. GP ligeramente plegado en forma de copa, vista polar distal oblicua; 4. SE y abertura. Bouteloua curtipendula. 5. GP plegado de forma irregular, vista polar distal; 6. SE y detalle de la abertura. Cenchrus myosuroides. 7. GP, vista polar distal oblicua; 8. SE. Dichanthium annulatum. 9. GP plegado, vista ecuatorial; 10. SE y abertura. Digitaria sanguinalis. 11. granos de polen plegados en forma irregular. 12. SE y abertura. Eragrostis lehmanniana. 13. GP, vista polar proximal; 14. SE y abertura. Panicum texanum. 15. granos de polen ligeramente plegados; 16. SE y abertura. Pennisetum ciliare. 17. GP totalmente plegado. 18. SE y apertura en un grano plegado. Poa pratensis. 19. GP plegado, vista polar distal oblicua, pared locular al fondo; 20. SE y abertura. Setaria liebmannii. 21. GP, vista polar distal; 22. SE de un GP totalmente plegado. Sorghum halepense. 23. GP ligeramente plegado, vista ecuatorial; 24. SE y abertura. 

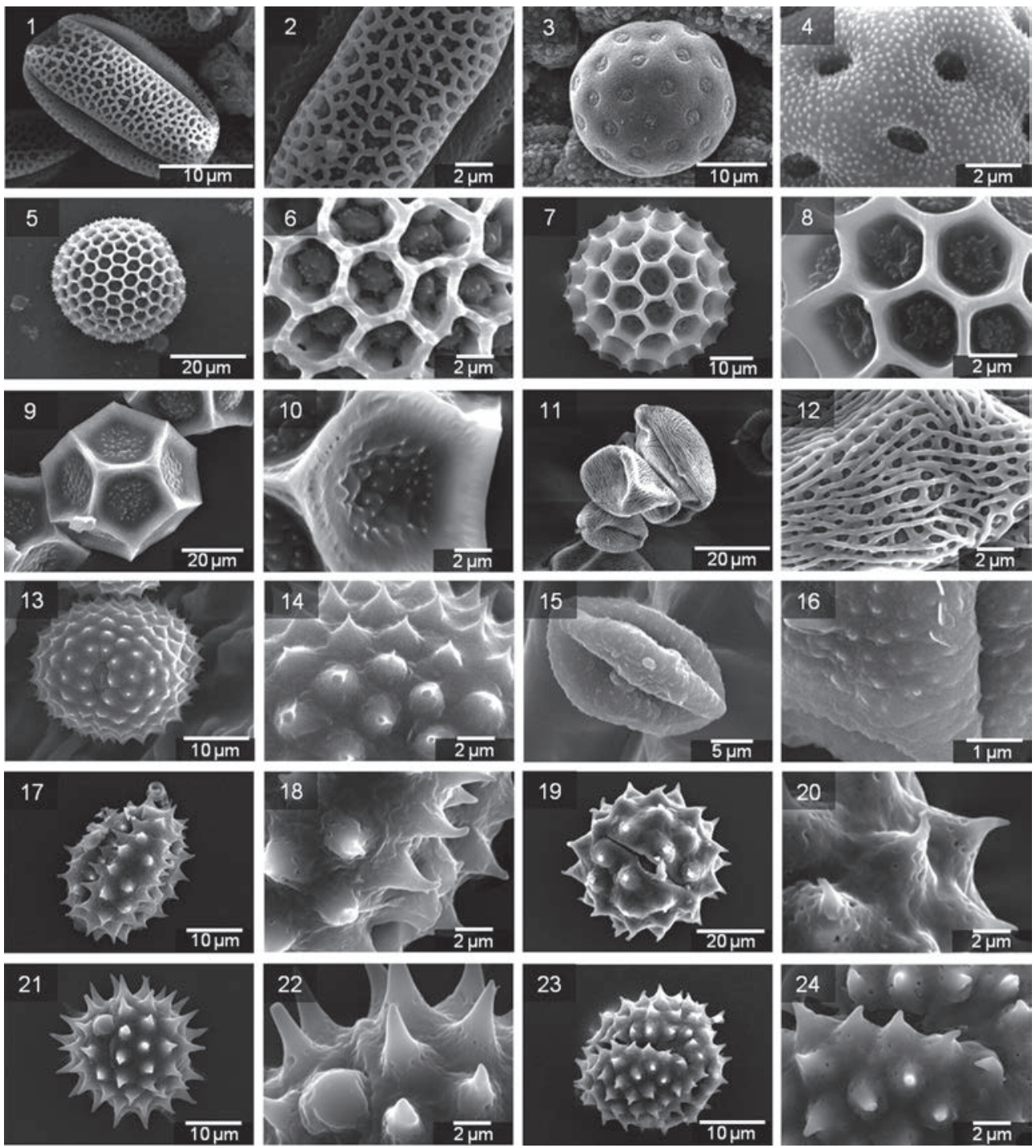

Lámina III. Sambucus nigra. 1. grano de polen (GP), vista ecuatorial; 2. superficie de la exina (SE). Amaranthus retroflexus. 3. GP; 4. SE. Froelichia arizonica. 5. GP; 6. SE. Gomphrena sonorae. 7. GP; 8. SE. Tidestromia lanuginosa. 9. GP; 10. SE. Schinus molle. 11. GP totalmente plegados; 12. SE. Ambrosia psilostachya. 13. GP, vista de la abertura; 14. SE. Artemisia frigida. 15. GP, vista ecuatorial oblicua; 16. SE. Aster ericoides. 17. GP, vista ecuatorial; 18. SE. Baileya multiradiata. 19. GP, vista ecuatorial; 20. SE. Bidens bigelovii. 21. GP; 22. SE. Conyza canadensis. 23. GP; 24. SE. 

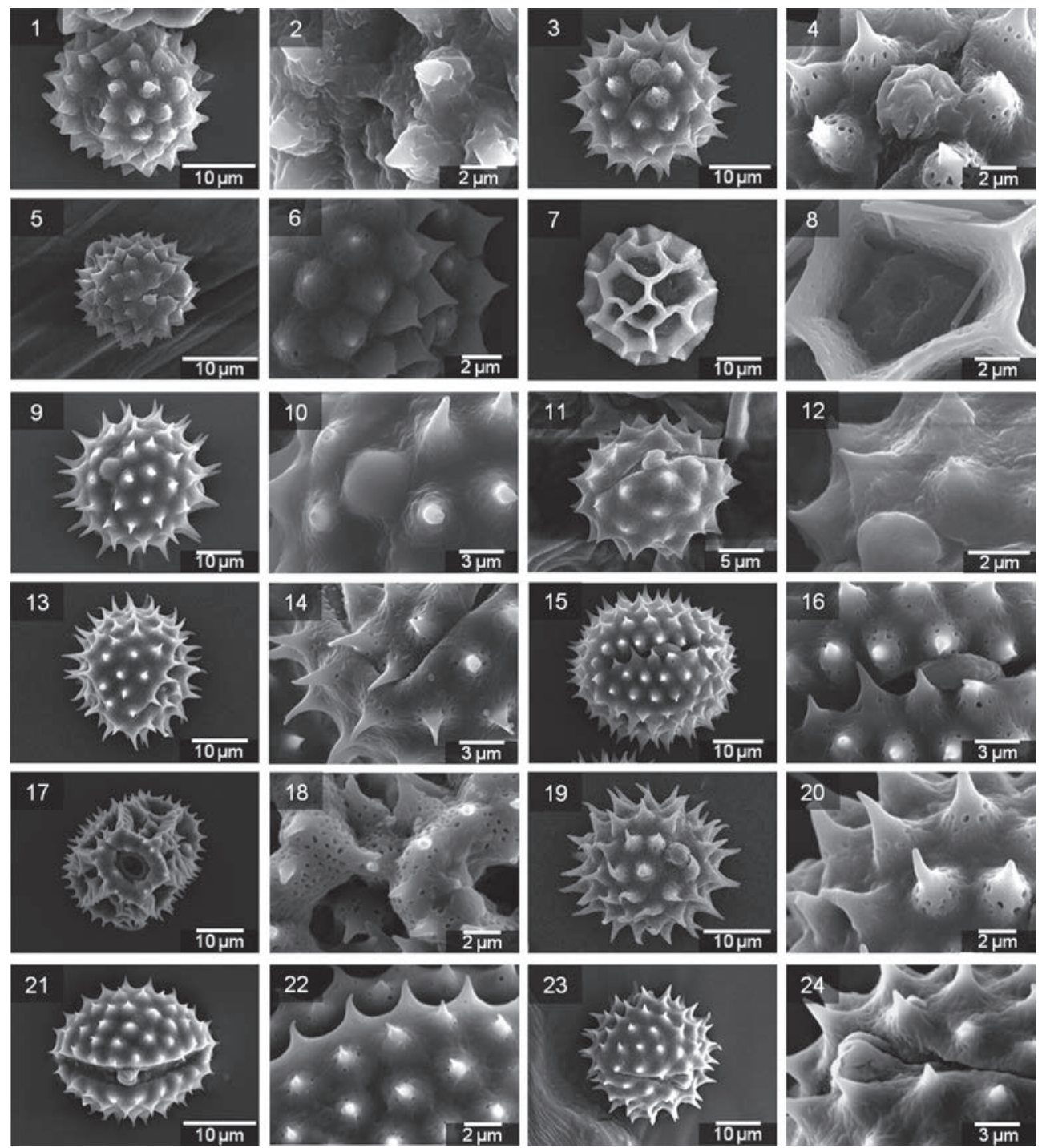

Lámina IV. Dyssodia pentachaeta. 1. grano de polen (GP); 2. superficie de la exina (SE). Dyssodia porophyllum. 3. GP, vista de la abertura; 4. SE y detalle de la abertura. Erigeron delphinifolius. 5. GP, vista polar oblicua; 6. SE. Gazania x splendens. 7. GP; 8. SE. Helianthus annuus. 9. GP, vista ecuatorial; 10. SE y detalle de la abertura. Hymenopappus scabiosaeus. 11. GP, vista ecuatorial; 12. SE. Melampodium hispidum. 13. GP, vista ecuatorial; 14. SE en el área polar. Tagetes patula. 15. GP, vista ecuatorial; 16. SE y detalle de la abertura. Taraxacum officinale. 17. GP, vista ecuatorial; 18. SE. Verbesina encelioides. 19. GP; 20. SE. Viguiera annua. 21. GP, vista ecuatorial; 22. SE. Zinnia grandiflora. 23. GP, vista polar oblicua; 24. SE y detalle de la abertura. 

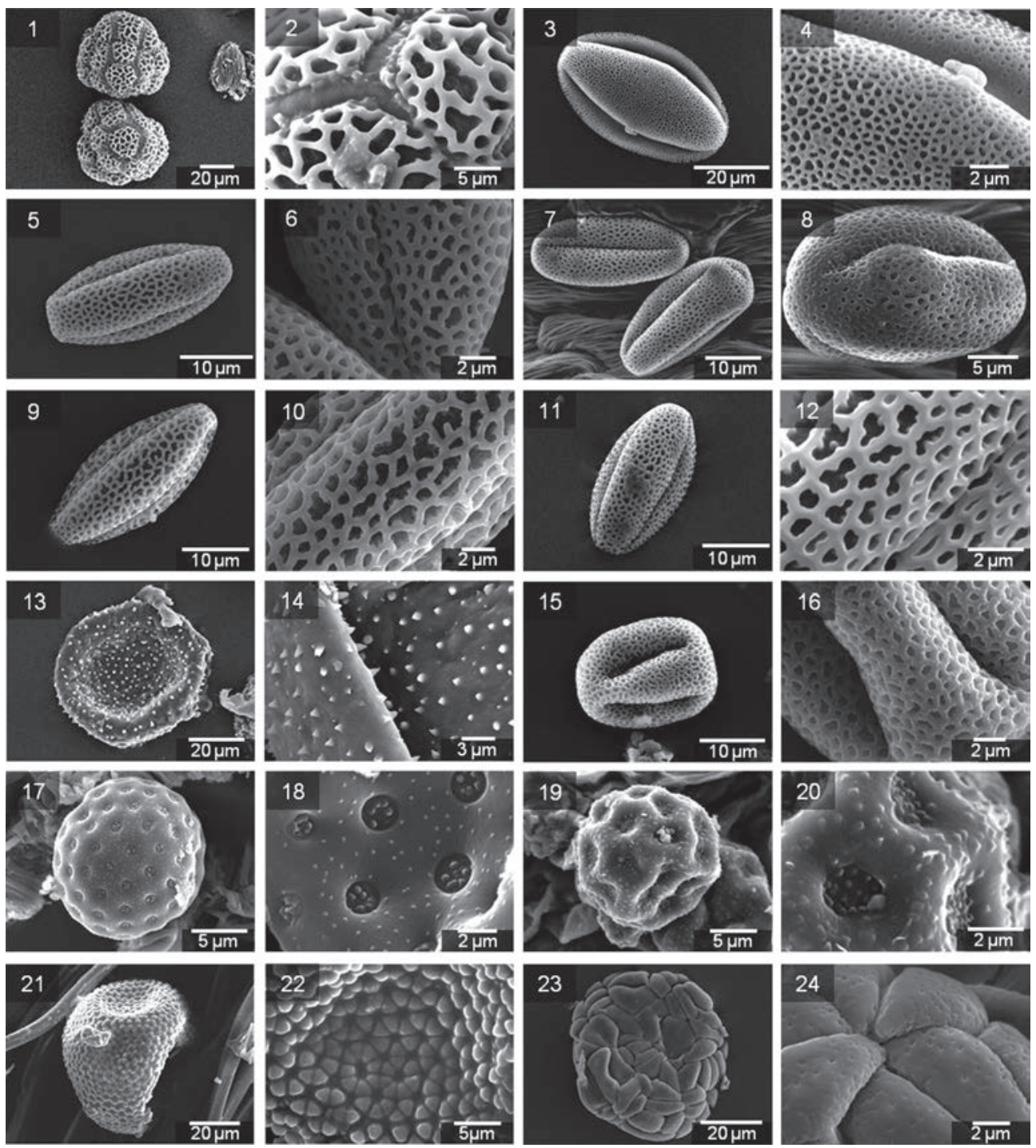

Lámina V. Chilopsis linearis. 1. granos de polen (GP); 2. superficie de la exina (SE). Tecoma stans. 3. GP, vista ecuatorial; 4. SE. Capsella bursa-pastoris. 5. GP, vista ecuatorial; 6. SE. Descurainia pinnata. 7. GP, vista ecuatorial y oblicua; 8. GP, vista polar oblicua. Lepidium virginicum. 9. GP, vista ecuatorial; 10. SE. Stanleya pinnata. 11. GP, vista ecuatorial; 12. SE. Abelia chinensis. 13. GP plegado en forma de copa; 14. SE. Casuarina equisetifolia. 15. GP, vista ecuatorial; 16. SE. Chenopodium murale. 17. GP; 18. SE y aberturas. Salsola iberica. 19. GP; 20. SE y aberturas. Croton pottsii. 21. GP; 22. SE mostrando el característico "patrón crotón”. Acacia farnesiana. 23. GP; 24. SE. 

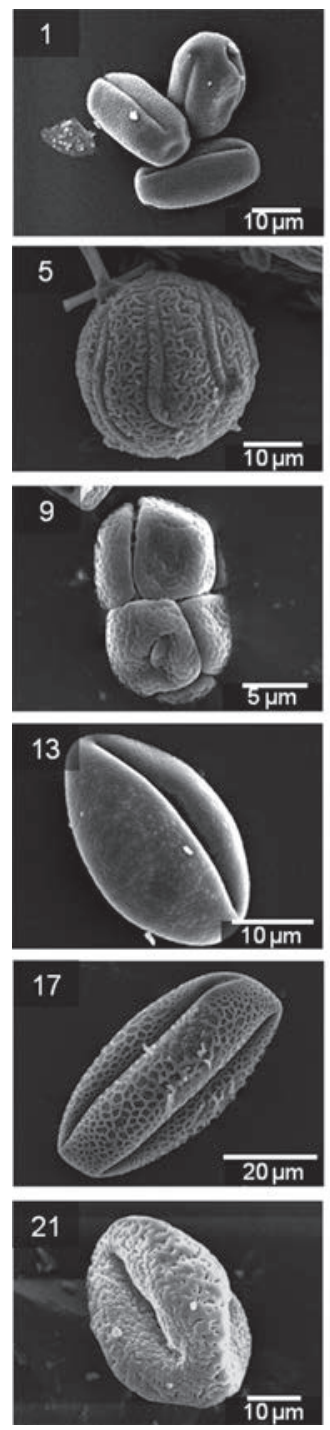
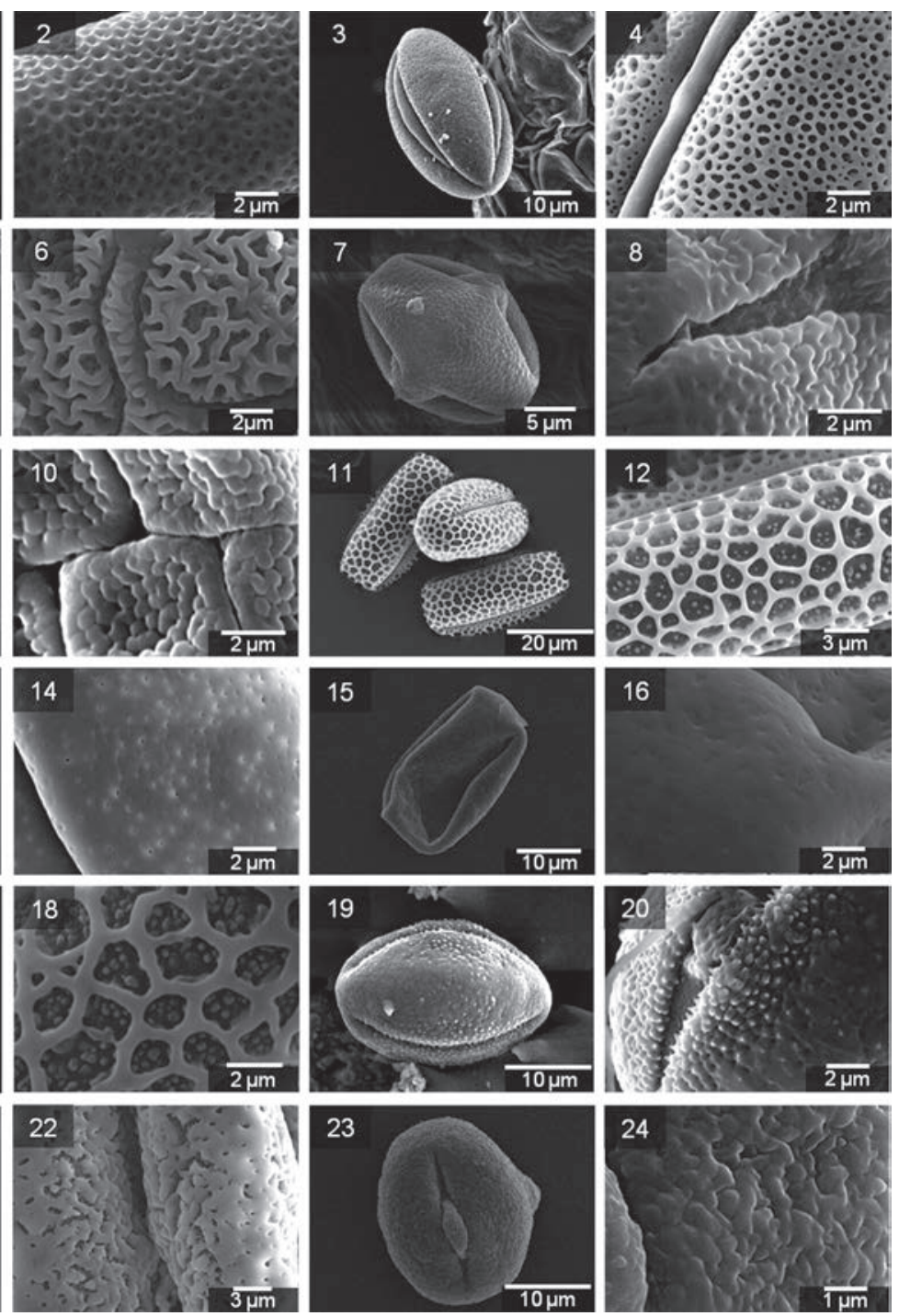
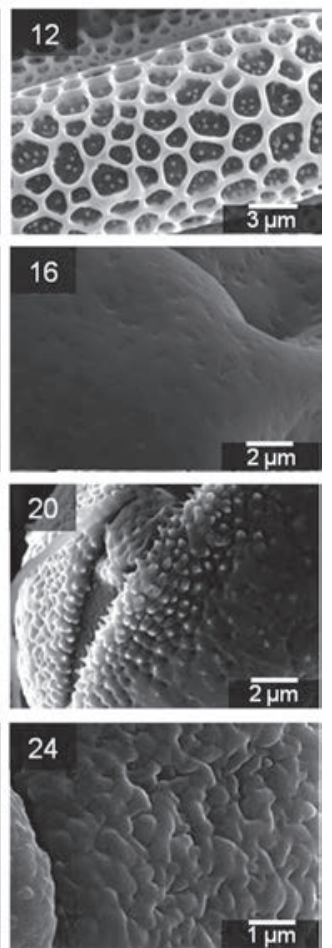

Lámina VI. Astragalus mollissimus. 1. grupo de granos de polen (GP), vista polar oblicua y ecuatorial; 2. superficie de la exina (SE). Astragalus nuttallianus. 3. GP, vista polar oblicua; 4. SE y abertura. Dalea neomexicana. 5. GP; 6. SE. Melilotus indicus. 7. GP, vista ecuatorial; 8. SE y abertura. Mimosa biuncifera. 9. GP; 10. SE. Parkinsonia aculeata. 11. grupo de GP, vista polar oblicua y ecuatorial; 12. SE. Prosopis glandulosa. 13. GP, vista ecuatorial; 14. SE. Robinia pseudoacacia. 15. GP totalmente plegado. 16. SE. Fouquieria splendens. 17. GP, vista ecuatorial; 18. SE. Cevallia sinuata. 19. GP, vista ecuatorial; 20. SE y detalle del área de la abertura. Lagerstroemia parviflora. 21. GP, vista polar oblicua; 22. SE. Punica granatum. 22. GP, vista ecuatorial; 23. SE. 

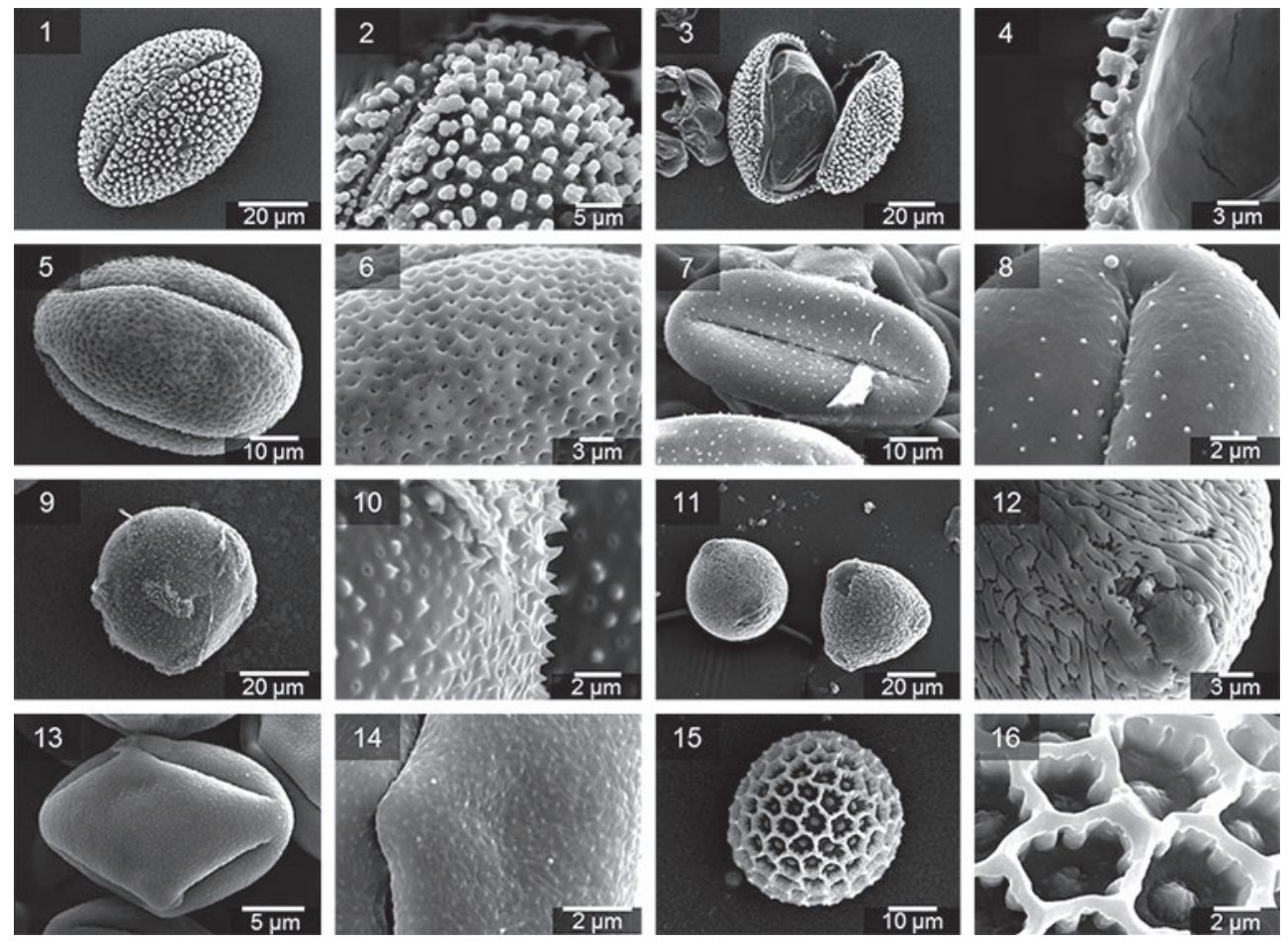

Lámina VII. Hibiscus hybridus. 1. grano de polen (GP); 2. superficie de la exina (SE) y espinas mayores de $10 \mu \mathrm{m}$. Sida procumbens. 3. GP; 4. SE. Sphaeralcea angustifolia. 5. GP; 6. GP mostrando rupturas en la exina. Proboscidea louisianica. 7. GP; 8. SE. Proboscidea fragrans. 9. GP; 10. SE. Melia azedarach. 11. GP, vista ecuatorial; 12. SE. Morus nigra. 13. GP plegado de forma irregular; 14. SE. Eucalyptus camaldulensis. 15. GP, vista polar oblicua; 16. SE. Acleisanthes longiflora. 17. grupo de GP; 18. SE y detalle de las aberturas. Boerhavia anisophylla. 19. GP; 20. SE. Boerhavia gracillima. 21. GP; 22. SE. Bougainvillea glabra. 23. GP; 24. SE. 

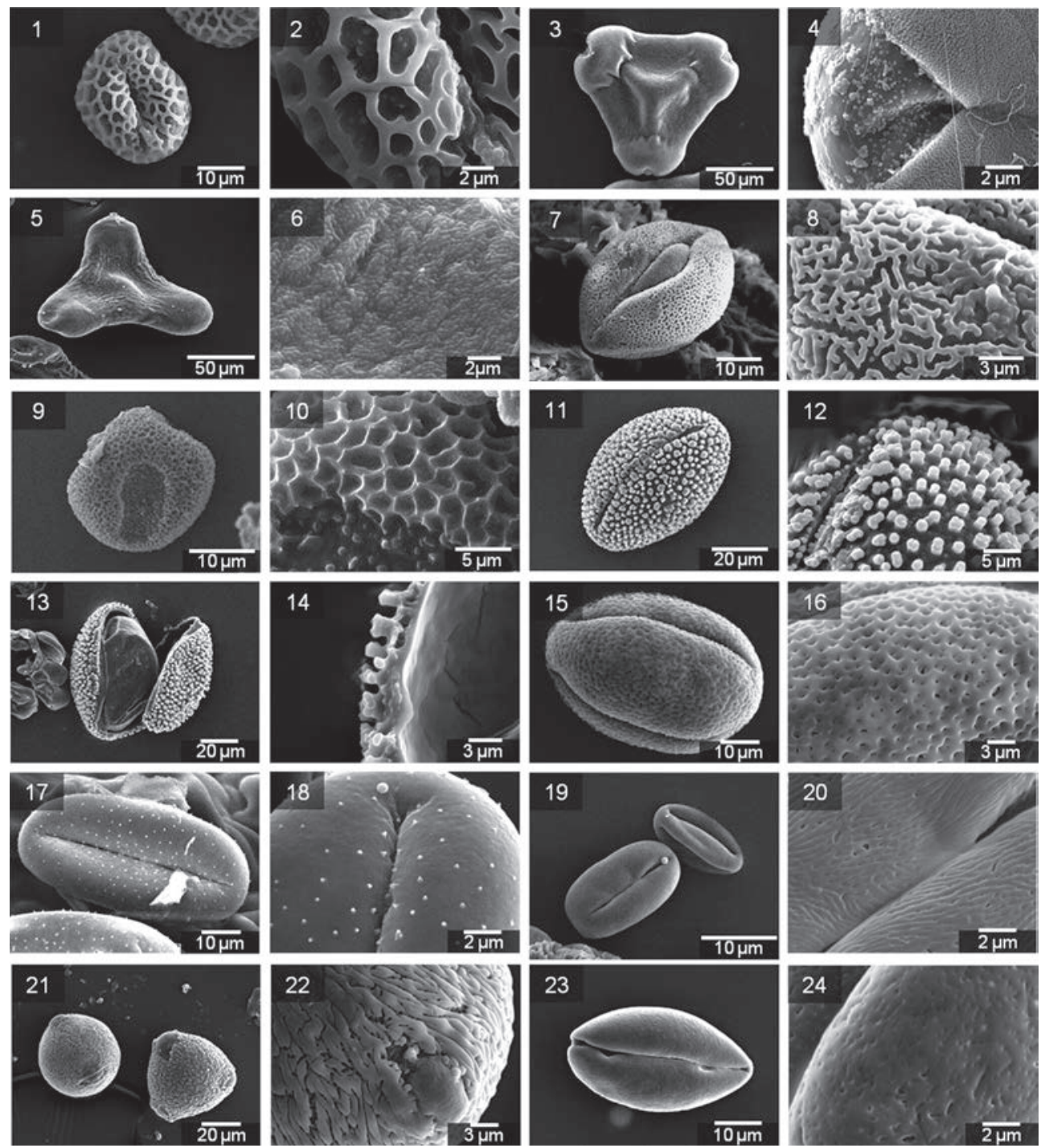

Lámina VIII. Ligustrum japonicum. 1. grano de polen (GP), vista ecuatorial; 2. superficie de la exina (SE). Oenothera serrula. 3. GP, vista ecuatorial; 4. SE y abertura. Oenothera gracilis. 5. GP, vista oblicua; 6. SE. Argemone ochroleuca. 7. GP, vista oblicua; 8. SE. Platanus orientalis. 9. GP, vista polar oblicua; 10. SE en área aledaña a la abertura. Plumbago scandens. 11. GP, vista ecuatorial; 12 . SE; 13. GP mostrando ruptura de la exina; 14 . detalles de exina, sección transversal. Antigonon leptopus. 15. GP, vista ecuatorial; 16. SE. Polygonum aviculare. 17. GP, vista ecuatorial; 18. SE. Pyracantha coccinea. 19. GP, vista ecuatorial; 20. SE. Datura stramonium. 21. GP; 22. SE y detalle de la abertura. Nicotiana glauca. 23. GP, vista ecuatorial oblicua; 24. SE. 

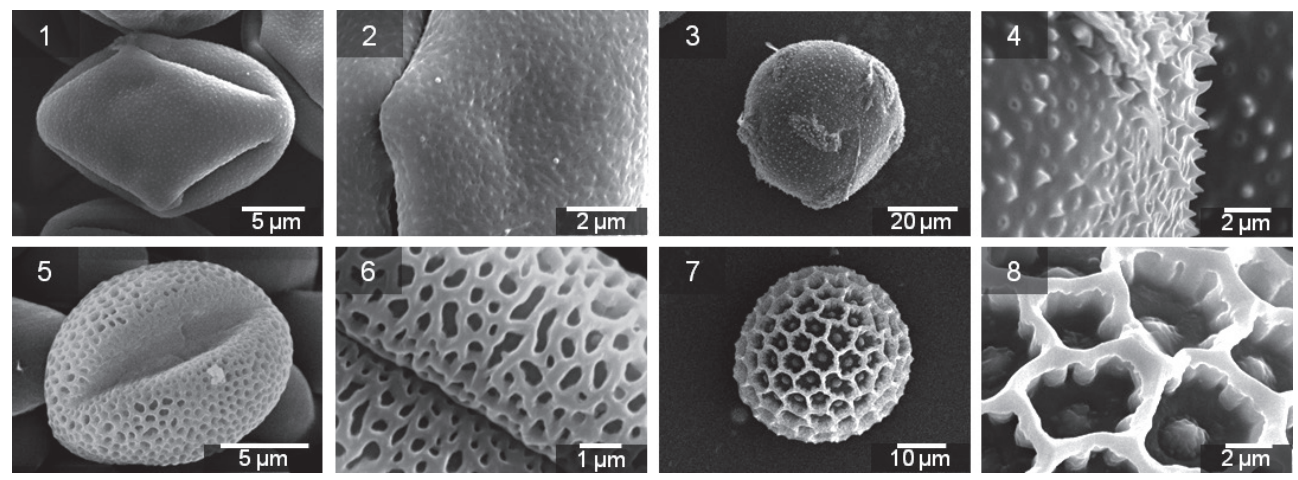

Lámina IX. Solanum rostratum. 1. grano de polen (GP), vista ecuatorial; 2. superficie de la exina (SE). Talinum aurantiacum. 3. GP; 4. SE. Tamarix gallica. 5. GP, vista ecuatorial; 6. SE. Tribulus terrestris. 7. GP; 8. SE.

\section{AGRADECIMIENTOS}

Los autores desean agradecer a Karla Campos-Venegas del Laboratorio Nacional de Nanotecnología del Centro de Investigación en Materiales Avanzados por su apoyo en la obtención de las imágenes del MEB.

\section{LITERATURA CITADA}

Adecanmbi, O. H. 2009. Pollen grains of Asteraceae and analogous echinate grains. Int. J. Bot. 5: 295-300.

Aguirre, A. y G. Muñoz. 2009. Identificación y análisis polínico de la vegetación de la ciudad de Chihuahua, México. Tesis de licenciatura. Facultad de Zootecnia y Ecología, Universidad Autónoma de Chihuahua. Chihuahua, México. 165 pp.

Anónimo. 2000. Normales climatológicas. Chihuahua, Chih. Período 1981-2000. Servicio Meteorológico Nacional. http://smn.cna.gob.mx/observatorios/historica/chihuahua. pdf. Fecha de acceso: Agosto de 2012.

Arreguín-Sánchez, M. L., R. Palacios; D. L. Quiroz-García y D. Ramos-Zamora. 1988. Morfología de los granos de polen de la familia Polygalaceae del Valle de México. Acta Bot. Mex. 4: 21-27.

Arreguín-Sánchez, M. L., D. L. Quiroz-García y R. Palacios-Chávez. 1990. Morfología de los granos de polen de las familias Aceraceae, Aquifoliaceae, Geraniaceae, Resedaceae, Sabiaceae y Saxifragaceae del Valle de México. Acta Bot. Mex. 10: 3-21.

Belmonte, J. y J. M. Roure. 2002. Introducción. In: Valero, A. L. y A. Cadahía (eds.). Polinosis, polen y alergia. MRA Ediciones S.L. Barcelona, España. pp. 7-16. 
Buchner, R. y M. Weber. 2012. PalDat- a palynological database: descriptions, illustrations, identification, and information retrieval. http://www.paldat.org. Society for the Promotion of Palynological Research in Austria; University of Vienna. Viena, Austria. Fecha de acceso: Agosto del 2012.

Erdtman, G. 1952. Pollen morphology and Plant Taxonomy: Angiosperms. Almqvist \& Wiksell. Stockholm, Suecia. 539 pp.

Estrada-Castillón, E. 1995. Flora de la cuenca de la laguna Babícora, municipios de Gómez Farías y Madera, Chihuahua. Tesis de maestría. Facultad de Zootecnia y Ecología, Universidad Autónoma de Chihuahua. Chihuahua, México. 127 pp.

Estrada-Castillón, E. y J. A. Villarreal-Quintanilla. 2010. Flora del centro del estado de Chihuahua, México. Acta Bot. Mex. 92: 51-118.

Goreti, M. y R. G. Alfaro. 2003. Contribución al conocimiento de la morfología de los granos de polen del género Bourreria P. Browne (Boraginaceae) de México. Polibotánica 16: $1-27$.

Halbriter, H., M. Weber, R. Zetter, A. Frosch-Radivo, R. Buchner y M. Hesse. 2007. PalDat- Illustrated handbook on pollen terminology. Society for the Promotion of Palynological Research in Austria. Viena, Austria. 70 pp.

Hesse, M., H. Halbritter, R. Zetter, M. Weber, R. Buchner, A. Frosch-Radivo y S. Ulrich. 2009. Pollen terminology. An illustrated handbook. Springer. Wien New York. Viena, Austria. 266 pp.

Katifori, E., S. Albenc, E. Cerdae, D. R. Nelsona y J. Dumaisf. 2010. Foldable structures and the natural design of pollen grains. PNAS 107: 7635-7639.

Lebgue, T. y A. Valerio. 1986. Manual para identificar las gramíneas de Chihuahua. Gobierno del Estado de Chihuahua. Chihuahua, México. 231 pp.

Lebgue, T., M. Sosa y R. Soto. 2005. La flora de las Barrancas del Cobre, Chihuahua, México. Ecol. Aplicada 4: 17-23.

Morais, M., L. Moreira, X. Feas y L. M. Estevinho. 2011. Honeybee-collected pollen from five Portuguese Natural Parks: Palynological origin, phenolic content, antioxidant properties and antimicrobial activity. Food Chem. Toxicol. 49: 1096-1101.

Palacios-Chávez, R., M. L. Arreguín-Sánchez y D. L. Quiroz-García. 1996. Morfología de las familias Acanthaceae, Vitaceae y Violaceae del Valle de México. Acta Bot. Mex. 34: $1-24$.

Pino, J. A. y M. J. Díez. 1993. Aportación a la palinología de plantas ornamentales de la ciudad de Sevilla. Lagascalia 17: 87-103.

Punt, W., P. P. Hoen, S. Blackmore, S. Nilsson y A. Le Thomas. 2007. Glossary of pollen and spore terminology. Rev. Palaebot. Palynol. 143: 1-81.

Quiroz-García, D. L., R. Palacios y M. L. Arreguín-Sánchez. 1997. Morfología de los granos de polen de la familia Boraginaceae de la estación de biología de Chamela, Jalisco, México. Acta Bot. Mex. 4: 37-53.

Quiroz-García, D. L., M. L. Arreguín-Sánchez y S. Antuna. 2002. Morfología de los granos de polen de la familia Polemoniaceae del estado de Querétaro, México. Acta Bot. Mex. 14: 57-66.

Quiroz-García, D. L. y M. L. Arreguín Sánchez. 2004. Morfología de los granos de polen de la tribu Potentilleae (Rosaceae) del Valle de México. Polibotánica 18: 87-97. 
Rodríguez-Villa, M. A. 2009. Desarrollo de una metodología para la recuperación de pólenes captados en filtros de fibra de vidrio por muestreo de alto volumen. Tesis de maestría. Centro de Investigación en Materiales Avanzados. Chihuahua, México. 49 pp.

Royo, M. H., A. Melgoza. 2001. Listado florístico del Campo Experimental La Campana y usos de su flora. Rev. Mex. Cienc. Pecuarias 39: 105-125.

Rzedowski, J. 1998. Diversidad y orígenes de la flora fanerogámica de México. In: Ramamoorthy, T. P., R. Bye, A. Lot y J. Fa (eds.). Diversidad biológica de México: Orígenes y distribución. Instituto de Biología, Universidad Nacional Autónoma de México. México, D.F., México. pp. 129-145.

Spellenberg, R., T. Lebgue y R. Corral-Díaz. 1996. Listados florísticos de México. XIII. A specimen-based, annotated checklist of the vascular plants of Parque Nacional "Cascada de Basaseachi” and adjacent areas, Chihuahua, México. Instituto de Biología, Universidad Autónoma de México. México, D.F., México. 77 pp.

Torres, L., S. Fuentes y A. Delgado. 2010. Estudio palinológico del género Macroptilium (Benth.) Urb. (Leguminosae) en México y Centroamérica. Acta Bot. Mex. 91: 51-69. Vega, J. 2008. Listado florístico del cañón del río Chuviscar. Tesis de licenciatura. Facultad de Zootecnia y Ecología, Universidad Autónoma de Chihuahua. Chihuahua, México. Villaseñor, J. L. 2004. Los géneros de plantas vasculares en México. Bol. Soc. Bot. Mex. 75: 105-135. 


\section{APÉNDICE}

Características palinológicas generales de los taxones colectados.

Tamaño: $\mathrm{P}$ = pequeño, $\mathrm{M}=$ mediano, $\mathrm{G}=\mathrm{Grande}, \mathrm{MG}=$ muy grande. Ornamentación: $\mathrm{Bc}=$ baculada, $\mathrm{Cl}=$ clavada, $\mathrm{Cr}=$ cristada, $\mathrm{Es}=$ estriada, $\mathrm{Eq}=$ equinada, $\mathrm{Fs}=$ fosulada, $\mathrm{Gm}=$ gemada, $\mathrm{Gr}=$ granulada, $\mathrm{m}=$ micro, $\mathrm{Lf}=$ lofada, $\mathrm{Pf}=$ perforada, $\mathrm{Ps}=$ Psilada, $\mathrm{Rg}=$ rugulada, $\mathrm{Rt}=$ reticulada, $\mathrm{Vg}=$ verrugada. Abertura: Inap = inaperturado, Lep = leptoma, $\mathrm{Mcp}=$ monocolpado, Pap = pantoaperturado, Pcp $=$ pantocolpado, Po $=$ porado, Ppo $=$ pantoporado, Slc $=$ sulcado, Tcp $=$ tricolpado, $\mathrm{Tcpr}=$ tricolporado, $\mathrm{Tpo}=$ triporado, $\mathrm{UOA}=$ ulcerado-operculado-anulado. Forma: Esf = esférico, FB = forma de bote, $\mathrm{FC}$ = forma de copa, $\mathrm{Obl}=$ oblado, Polig $=$ poligonal; Prol $=$ prolado, Triang $=$ triangular. Plegamiento: $\mathrm{Ab}-\mathrm{Hn}=$ aberturas hundidas, No-Plg = no plegado, Plg-Irr = plegamiento irregular.

\begin{tabular}{|c|c|c|c|c|c|c|c|}
\hline Taxon & $\begin{array}{l}\text { Tama- } \\
\text { ño }\end{array}$ & $\begin{array}{c}\text { Ornamen- } \\
\text { tación }\end{array}$ & $\begin{array}{l}\text { Tipo de } \\
\text { apertura }\end{array}$ & $\begin{array}{c}\text { Forma } \\
\text { granos } \\
\text { hidratados }\end{array}$ & $\begin{array}{c}\text { Forma } \\
\text { granos } \\
\text { secos }\end{array}$ & $\begin{array}{l}\text { Plega- } \\
\text { miento }\end{array}$ & $\begin{array}{l}\text { Lámina, } \\
\text { Núm. de } \\
\text { figura }\end{array}$ \\
\hline
\end{tabular}

\section{Gimnospermas}

Pinaceae

Pinus halepensis Mill. $\quad$ M $\quad$ Vg $\quad$ Lep $\quad$ Irr $\quad$ Irr Ab-Hn I, 1-2

\section{Angiospermas}

\section{Monocotiledóneas}

Agavaceae

Yucca sp.

M Ps $\quad$ Mcp $\quad$ Esf $\quad$ Prol Ab-Hn I, 3-4

Cyperaceae

Cyperus esculentus L. $\quad$ M $\quad \mathrm{mEq} \quad$ Ppo $\quad$ Irr $\quad$ Irr $\quad$ Ab-Hn I, 5-6

Iridaceae

\section{Hermodactylus} tuberosus (L.) Mill.

G Rt $\quad$ Slc $\quad$ Prol $\quad$ FB $\quad$ Ab-Hn II, 7-8

Poaceae

\begin{tabular}{|c|c|c|c|c|c|c|c|}
\hline $\begin{array}{l}\text { Aristida adscensionis } \\
\quad \text { L. }\end{array}$ & M & $\mathrm{mEq}$ & UOA & Esf & Esf & Plg-Irr & II, 1-2 \\
\hline $\begin{array}{l}\text { Aristida pansa } \\
\quad \text { Wooton \& Standl. }\end{array}$ & $\mathrm{M}$ & Gr & UOA & Esf & Esf & Plg-Irr & II, 3-4 \\
\hline $\begin{array}{l}\text { Bouteloua } \\
\text { curtipendula }\end{array}$ & $\mathrm{M}$ & Fs, Gr & UOA & Esf & Esf & Plg-Irr & II, 5-6 \\
\hline
\end{tabular}

(Michx.) Torr. 
Apéndice. Continuación.

\begin{tabular}{|c|c|c|c|c|c|c|c|}
\hline Taxon & $\begin{array}{l}\text { Tama- } \\
\text { ño }\end{array}$ & $\begin{array}{l}\text { Ornamen- } \\
\text { tación }\end{array}$ & $\begin{array}{l}\text { Tipo de } \\
\text { apertura }\end{array}$ & $\begin{array}{c}\text { Forma } \\
\text { granos } \\
\text { hidratados } \\
\end{array}$ & $\begin{array}{l}\text { Forma } \\
\text { granos } \\
\text { secos } \\
\end{array}$ & $\begin{array}{l}\text { Plega- } \\
\text { miento }\end{array}$ & $\begin{array}{l}\text { Lámina, } \\
\text { Núm. de } \\
\text { figura }\end{array}$ \\
\hline $\begin{array}{l}\text { Cenchrus } \\
\text { myosuroides Kunth }\end{array}$ & $\mathrm{M}$ & Fs, Gr & UOA & Esf & Esf & Plg-Irr & II, 7-8 \\
\hline $\begin{array}{l}\text { Dichanthium } \\
\text { annulatum (Forssk.) } \\
\text { Stapf }\end{array}$ & M & Fs, Gr & UOA & Esf & Esf & Plg-Irr & II, 9-10 \\
\hline $\begin{array}{l}\text { Digitaria sanguinalis } \\
\text { (L.) Scop. }\end{array}$ & M & Fs, Gr & UOA & Esf & Esf & Plg-Irr & $\begin{array}{l}\text { II, } 11- \\
12\end{array}$ \\
\hline $\begin{array}{l}\text { Eragrostis } \\
\quad \text { lehmanniana Nees }\end{array}$ & M & Fs, Gr & UOA & Esf & Esf & Plg-Irr & $\begin{array}{l}\text { II, } 13- \\
14\end{array}$ \\
\hline $\begin{array}{l}\text { Panicum texanum } \\
\text { Buckley }\end{array}$ & M & Fs, Gr & UOA & Esf & Esf & Plg-Irr & $\begin{array}{l}\text { II, } 15- \\
16\end{array}$ \\
\hline $\begin{array}{l}\text { Pennisetum ciliare } \\
\text { (L.) Link }\end{array}$ & M & Fs, Gr & UOA & Esf & Esf & Plg-Irr & $\begin{array}{l}\text { II, } 17- \\
18\end{array}$ \\
\hline Poa pratensis L. & M & Fs, Gr & UOA & Esf & Esf & Plg-Irr & $\begin{array}{l}\text { II, 19- } \\
20\end{array}$ \\
\hline $\begin{array}{l}\text { Setaria liebmannii E. } \\
\text { Fourn. }\end{array}$ & M & Fs, Gr & UOA & Esf & Esf & Plg-Irr & $\begin{array}{l}\text { II, } 21- \\
22\end{array}$ \\
\hline $\begin{array}{l}\text { Sorghum halepense } \\
\text { (L.) Pers. }\end{array}$ & M & Gr & UOA & Esf & Esf & Plg-Irr & $\begin{array}{l}\text { II, 23- } \\
24\end{array}$ \\
\hline
\end{tabular}

\section{Dicotiledóneas}

Adoxaceae

Sambucus nigra L.

P $\quad$ Rt

Rt Tpo

Esf Prol

Ab-Hn III, 1-2

Amaranthaceae

Amaranthus

P $\quad$ mEq Ppo

Esf

Esf

Algunas

III, 3-4

retroflexus L.

veces

ligera FC

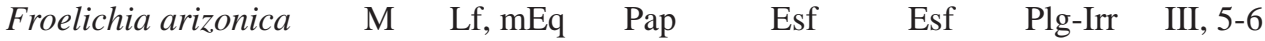

Thornb. ex Standl.

Gomphrena sonorae

Torr.

Tidestromia

M

Lf $\quad$ Pap

Esf

Esf

Plg-Irr

III, 7-8

Tidestromia

M Lf, mEq

Pap

Polig

Polig No-Plg

III, lanuginosa (Nutt.)

Standl.

Anacardiaceae

Schinus molle L.

M Es, Rt Tcp

Esf Prol

Ab-Hn III, 11- 
Apéndice. Continuación.

\begin{tabular}{|c|c|c|c|c|c|c|c|}
\hline Taxon & $\begin{array}{l}\text { Tama- } \\
\text { ño }\end{array}$ & $\begin{array}{l}\text { Ornamen- } \\
\text { tación }\end{array}$ & $\begin{array}{l}\text { Tipo de } \\
\text { apertura }\end{array}$ & $\begin{array}{c}\text { Forma } \\
\text { granos } \\
\text { hidratados }\end{array}$ & $\begin{array}{l}\text { Forma } \\
\text { granos } \\
\text { secos } \\
\end{array}$ & $\begin{array}{l}\text { Plega- } \\
\text { miento }\end{array}$ & $\begin{array}{l}\text { Lámina, } \\
\text { Núm. de } \\
\text { figura }\end{array}$ \\
\hline \multicolumn{8}{|l|}{ Asteraceae } \\
\hline $\begin{array}{l}\text { Ambrosia } \\
\text { psilostachya DC. }\end{array}$ & $\mathrm{P}$ & $\mathrm{Eq}$ & Тср & Esf & Irr & Ab-Hn & $\begin{array}{c}\text { III, } 13- \\
14\end{array}$ \\
\hline $\begin{array}{l}\text { Artemisia frigida } \\
\text { Willd. }\end{array}$ & $\mathrm{P}$ & $\mathrm{mEq}, \mathrm{Gr}$ & Tcpr & Esf & Prol & Ab-Hn & $\begin{array}{c}\text { III, } 15- \\
16\end{array}$ \\
\hline Aster ericoides L. & $\mathrm{P}$ & $\mathrm{Eq}$ & Tcpr & Esf & Prol & Ab-Hn & $\begin{array}{c}\text { III, } 17- \\
18\end{array}$ \\
\hline $\begin{array}{c}\text { Baileya multiradiata } \\
\text { Harv. \& A. Gray }\end{array}$ & M & $\mathrm{Eq}, \mathrm{Pf}$ & Tcpr & Esf & Prol & Ab-Hn & $\begin{array}{l}\text { III, 19- } \\
20\end{array}$ \\
\hline $\begin{array}{l}\text { Bidens bigelovii A. } \\
\text { Gray }\end{array}$ & M & Eq, Pf & Tcpr & Esf & Prol & $\mathrm{Ab}-\mathrm{Hn}$ & $\begin{array}{c}\text { III, } 21- \\
22\end{array}$ \\
\hline $\begin{array}{l}\text { Conyza canadensis } \\
\text { (L.) Cronquist }\end{array}$ & M & Eq, Pf & Tcpr & Esf & Prol & Ab-Hn & $\begin{array}{c}\text { III, } 23- \\
24\end{array}$ \\
\hline $\begin{array}{l}\text { Dyssodia pentachaeta } \\
\text { (DC.) B.L. Rob. }\end{array}$ & $\mathrm{P}$ & Eq, Pf & Tcpr & Esf & Prol & Ab-Hn & IV, 1-2 \\
\hline $\begin{array}{l}\text { Dyssodia } \\
\text { porophyllum (Cav.) } \\
\text { Cav. }\end{array}$ & $\mathrm{P}$ & Eq, Pf & Tcpr & Esf & Prol & Ab-Hn & IV, 3-4 \\
\hline $\begin{array}{l}\text { Erigeron } \\
\text { delphinifolius } \\
\text { Willd. }\end{array}$ & M & Eq, Pf & Tcpr & Esf & Prol & Ab-Hn & IV, 5-6 \\
\hline $\begin{array}{l}\text { Gazania x splendens } \\
\text { Hort.Agl. }\end{array}$ & M & Lf & Tcpr & Esf & Esf & No-Plg & IV, 7-8 \\
\hline Helianthus annuus L. & $\mathrm{P}$ & Eq, Pf & Tcpr & Esf & Prol & Ab-Hn & $\begin{array}{l}\text { IV, } \\
9-10\end{array}$ \\
\hline $\begin{array}{l}\text { Hymenopappus } \\
\text { scabiosaeus L'Hér. }\end{array}$ & $\mathrm{P}$ & Eq, Pf & Tcpr & Esf & Prol & Ab-Hn & $\begin{array}{c}\text { IV, } 11- \\
12\end{array}$ \\
\hline $\begin{array}{l}\text { Melampodium } \\
\text { hispidum Kunth }\end{array}$ & M & Eq, Pf & Tcpr & Esf & Prol & Ab-Hn & $\begin{array}{c}\text { IV, } 13- \\
14\end{array}$ \\
\hline Tagetes patula L. & M & Eq, Pf & Tcpr & Esf & Prol & Ab-Hn & $\begin{array}{c}\text { IV, } 15- \\
16\end{array}$ \\
\hline $\begin{array}{l}\text { Taraxacum officinale } \\
\text { F.H. Wigg. }\end{array}$ & M & Eq, Pf & Tcpr & Esf & Esf & No-Plg & $\begin{array}{c}\text { IV, } 17- \\
18\end{array}$ \\
\hline $\begin{array}{l}\text { Verbesina encelioides } \\
\text { (Cav.) Benth. \& } \\
\text { Hook. ex A. Gray }\end{array}$ & M & Eq, Pf & Tcpr & Esf & Prol & Ab-Hn & $\begin{array}{l}\text { IV, } 19- \\
20\end{array}$ \\
\hline
\end{tabular}


Apéndice. Continuación.

\begin{tabular}{|c|c|c|c|c|c|c|c|}
\hline Taxon & $\begin{array}{l}\text { Tama- } \\
\text { ño }\end{array}$ & $\begin{array}{l}\text { Ornamen- } \\
\text { tación }\end{array}$ & $\begin{array}{l}\text { Tipo de } \\
\text { apertura }\end{array}$ & $\begin{array}{c}\text { Forma } \\
\text { granos } \\
\text { hidratados } \\
\end{array}$ & $\begin{array}{c}\text { Forma } \\
\text { granos } \\
\text { secos } \\
\end{array}$ & $\begin{array}{l}\text { Plega- } \\
\text { miento }\end{array}$ & $\begin{array}{l}\text { Lámina, } \\
\text { Núm. de } \\
\text { figura }\end{array}$ \\
\hline $\begin{array}{l}\text { Viguiera annua (M.E. } \\
\text { Jones) S.F. Blake }\end{array}$ & $\mathrm{M}$ & Eq, Pf & Tcpr & Esf & Prol & Ab-Hn & $\begin{array}{l}\text { IV, 21- } \\
22\end{array}$ \\
\hline $\begin{array}{l}\text { Zinnia grandiflora } \\
\text { Nutt. }\end{array}$ & M & $\mathrm{Eq}, \mathrm{Pf}$ & Tcpr & Esf & Prol & Ab-Hn & $\begin{array}{l}\text { IV, 23- } \\
24\end{array}$ \\
\hline \multicolumn{8}{|l|}{ Bignoniaceae } \\
\hline $\begin{array}{l}\text { Chilopsis linearis } \\
\text { (Cav.) Sweet }\end{array}$ & M & Rt & Рср & Irr & Irr & No-Plg & $\mathrm{V}, 1-2$ \\
\hline $\begin{array}{l}\text { Tecoma stans (L.) } \\
\text { Juss. ex Kunth }\end{array}$ & M & $\mathrm{mRt}$ & Tcpr & Esf & Prol & Ab-Hn & V, 3-4 \\
\hline \multicolumn{8}{|l|}{ Brassicaceae } \\
\hline $\begin{array}{l}\text { Capsella bursa- } \\
\text { pastoris (L.) Medik. }\end{array}$ & $\mathrm{P}$ & Rt & Тср & Esf & Prol & Ab-Hn & V, 5-6 \\
\hline $\begin{array}{l}\text { Descurainia pinnata } \\
\text { (Walter) Britton }\end{array}$ & M & Rt & Тср & Esf & Prol & Ab-Hn & V, 7-8 \\
\hline $\begin{array}{l}\text { Lepidium virginicum } \\
\text { L. }\end{array}$ & M & Rt & Тср & Prol & Prol & Ab-Hn & V, 9-10 \\
\hline $\begin{array}{l}\text { Stanleya pinnata } \\
\text { (Pursh) Britton }\end{array}$ & $\mathrm{P}$ & Rt & Тср & Esf & Prol & Ab-Hn & $\begin{array}{c}\mathrm{V}, 11- \\
12\end{array}$ \\
\hline \multicolumn{8}{|l|}{ Caprifoliaceae } \\
\hline $\begin{array}{l}\text { Abelia chinensis R. } \\
\text { Br. }\end{array}$ & G & $\mathrm{mEq}$ & Tcpr & Esf & Irr & FC & $\begin{array}{l}\mathrm{V}, 13- \\
14\end{array}$ \\
\hline \multicolumn{8}{|l|}{ Casuarinaceae } \\
\hline $\begin{array}{l}\text { Casuarina } \\
\text { equisetifolia L. } \\
\text { Chenopodiaceae }\end{array}$ & $\mathrm{P}$ & Rt & Tpo & Esf & Prol & Ab-Hn & $\begin{array}{l}\mathrm{V}, 15- \\
16\end{array}$ \\
\hline $\begin{array}{l}\text { Chenopodium murale } \\
\text { L. }\end{array}$ & M & $\mathrm{mEq}$ & Ppo & Esf & Irr & $\begin{array}{l}\text { Plg-Irr, } \\
\text { frecuente } \\
\text { FC }\end{array}$ & $\begin{array}{l}\text { V, 17- } \\
18\end{array}$ \\
\hline $\begin{array}{l}\text { Salsola iberica } \\
\text { (Sennen \& Pau) } \\
\text { Botsch. ex. Czerep. }\end{array}$ & $\mathrm{P}$ & $\mathrm{mEq}$ & Ppo & Esf & Irr & $\begin{array}{l}\text { Plg-Irr, } \\
\text { frecuente } \\
\text { FC }\end{array}$ & $\begin{array}{l}\text { V, 19- } \\
20\end{array}$ \\
\hline Euphorbiaceae & & & & & & & \\
\hline $\begin{array}{l}\text { Croton pottsii } \\
\text { (Klotzsch) Müll. } \\
\text { Arg. }\end{array}$ & G & $\begin{array}{l}\text { Rt-Cr, } \\
\text { patrón } \\
\text { croton }\end{array}$ & Inap & Esf & Prol & Ligera FC & $\begin{array}{l}\mathrm{V}, 21- \\
22\end{array}$ \\
\hline
\end{tabular}


Apéndice. Continuación.

\begin{tabular}{|c|c|c|c|c|c|c|c|}
\hline Taxon & $\begin{array}{l}\text { Tama- } \\
\text { ño }\end{array}$ & $\begin{array}{l}\text { Ornamen- } \\
\text { tación }\end{array}$ & $\begin{array}{l}\text { Tipo de } \\
\text { apertura }\end{array}$ & $\begin{array}{c}\text { Forma } \\
\text { granos } \\
\text { hidratados }\end{array}$ & $\begin{array}{c}\text { Forma } \\
\text { granos } \\
\text { secos }\end{array}$ & $\begin{array}{l}\text { Plega- } \\
\text { miento }\end{array}$ & $\begin{array}{c}\text { Lámina, } \\
\text { Núm. de } \\
\text { figura }\end{array}$ \\
\hline \multicolumn{8}{|l|}{ Fabaceae } \\
\hline $\begin{array}{l}\text { Acacia farnesiana } \\
\text { (L.) Willd. }\end{array}$ & M & Ps & Inap & Esf & Circ & Ab-Hn & $\begin{array}{c}\mathrm{V}, 23- \\
24\end{array}$ \\
\hline $\begin{array}{l}\text { Astragalus } \\
\quad \text { mollissimus Torr. }\end{array}$ & M & Rt, Pf & Tcpr & Prol & Prol & Ab-Hn & VI, 1-2 \\
\hline $\begin{array}{l}\text { Astragalus } \\
\text { nuttallianus DC. }\end{array}$ & G & Rt, Pf & Tcpr & Prol & Prol & Ab-Hn & VI, 3-4 \\
\hline $\begin{array}{l}\text { Dalea neomexicana } \\
\text { (A. Gray) Cory }\end{array}$ & M & Rt & Pap & Esf & Esf & Plg-Irr & VI, 5-6 \\
\hline $\begin{array}{l}\text { Melilotus indicus (L.) } \\
\quad \text { All. }\end{array}$ & M & Rt & Tcpr & Prol & Prol & Ab-Hn & VI, 7-8 \\
\hline $\begin{array}{l}\text { Mimosa biuncifera } \\
\text { Benth. }\end{array}$ & $\mathrm{P}$ & Fs & Inap & Obl & Obl & No-Plg & $\begin{array}{l}\text { VI, } \\
9-10\end{array}$ \\
\hline $\begin{array}{l}\text { Parkinsonia aculeata } \\
\text { L. }\end{array}$ & M & Rt & Tcpr & Esf & Prol & Ab-Hn & $\begin{array}{c}\text { VI, } 11- \\
12\end{array}$ \\
\hline $\begin{array}{l}\text { Prosopis glandulosa } \\
\text { Torr. }\end{array}$ & $\mathrm{P}$ & Ps & Tcpr & Esf & Prol & Ab-Hn & $\begin{array}{c}\text { VI, } 13- \\
14\end{array}$ \\
\hline $\begin{array}{l}\text { Robinia pseudoacacia } \\
\text { L. }\end{array}$ & M & $\mathrm{Pf}$ & Тср & Esf & Prol & Ab-Hn & $\begin{array}{c}\text { VI, 15- } \\
16\end{array}$ \\
\hline
\end{tabular}

Fouquieriaceae

Fouquieria splendens $\quad \mathrm{M} \quad \mathrm{Rt} \quad$ Tcpr $\quad$ Esf $\quad$ Prol Ab-Hn VI, 17Engelm.

Mt Tepr 18

Loasaceae

Cevallia sinuata Lag. $\quad$ M $\quad$ Ps, mEq Tcpr Prol Prol Ab-Hn VI, 19-

Lythraceae

\begin{tabular}{|c|c|c|c|c|c|c|c|}
\hline $\begin{array}{l}\text { Lagerstroemia } \\
\text { parviflora } \text { Roxb. } \\
\text { Malvaceae }\end{array}$ & M & Fs & Tcpr & Esf & Prol & Ab-Hn & $\begin{array}{l}\text { VI, 21- } \\
22\end{array}$ \\
\hline $\begin{array}{l}\text { Hibiscus hybridus F. } \\
\text { Dietr. }\end{array}$ & $\mathrm{G}$ & $\mathrm{Eq}, \mathrm{Gr}$ & Ppo & Esf & Esf & FC & $\begin{array}{l}\text { VII, } \\
1-2\end{array}$ \\
\hline Sida procumbens Sw. & G & $\mathrm{Eq}, \mathrm{Vg}$ & Po & Esf & Esf & $\mathrm{FC}$ & $\begin{array}{l}\text { VII, } \\
3-4\end{array}$ \\
\hline $\begin{array}{l}\text { Sphaeralcea } \\
\quad \text { angustifolia (Cav.) }\end{array}$ & M & $\mathrm{Eq}, \mathrm{Gr}$ & Po & Esf & Esf & No-Plg & $\begin{array}{l}\text { VII, } \\
5-6\end{array}$ \\
\hline
\end{tabular}


Apéndice. Continuación.

\begin{tabular}{|c|c|c|c|c|c|c|c|}
\hline Taxon & $\begin{array}{l}\text { Tama- } \\
\text { ño }\end{array}$ & $\begin{array}{l}\text { Ornamen- } \\
\text { tación }\end{array}$ & $\begin{array}{l}\text { Tipo de } \\
\text { apertura }\end{array}$ & $\begin{array}{c}\text { Forma } \\
\text { granos } \\
\text { hidratados } \\
\end{array}$ & $\begin{array}{l}\text { Forma } \\
\text { granos } \\
\text { secos } \\
\end{array}$ & $\begin{array}{l}\text { Plega- } \\
\text { miento }\end{array}$ & $\begin{array}{l}\text { Lámina, } \\
\text { Núm. de } \\
\text { figura }\end{array}$ \\
\hline \multicolumn{8}{|l|}{ Martyniaceae } \\
\hline $\begin{array}{l}\text { Proboscidea fragrans } \\
\text { (Lindl.) Decne. }\end{array}$ & G & Rt & Inap & Esf & Esf & No-Plg & $\begin{array}{l}\text { VII, } \\
9-10\end{array}$ \\
\hline $\begin{array}{l}\text { Proboscidea louisiana } \\
\text { (Mill.) Thell. }\end{array}$ & G & Rt & Inap & Esf & Esf & No-Plg & $\begin{array}{l}\text { VII, } \\
7-8\end{array}$ \\
\hline Meliaceae & & & & & & & \\
\hline Melia azedarach L. & M & Ps & Tcpr & Esf & Prol & Ab-Hn & $\begin{array}{l}\text { VII, } \\
11-12\end{array}$ \\
\hline \multicolumn{8}{|l|}{ Moraceae } \\
\hline Morus nigra L. & M & $\mathrm{mEq}$ & Тро & Esf & Irr & Plg-Irr & $\begin{array}{l}\text { VII, } \\
13-14\end{array}$ \\
\hline \multicolumn{8}{|l|}{ Myrtaceae } \\
\hline $\begin{array}{l}\text { Eucalyptus } \\
\text { camaldulensis } \\
\text { Dehnh. }\end{array}$ & M & Fs & Tcpr & Esf & Triang & $\begin{array}{l}\text { Área Inap } \\
\text { Hn }\end{array}$ & $\begin{array}{l}\text { VII, } \\
15-16\end{array}$ \\
\hline \multicolumn{8}{|l|}{ Nyctaginaceae } \\
\hline $\begin{array}{l}\text { Acleisanthes } \\
\quad \text { longiflora A. Gray }\end{array}$ & MG & $\mathrm{mEq}$ & Ppo & Esf & Esf & $\mathrm{FC}$ & $\begin{array}{l}\text { VII, } \\
17-18\end{array}$ \\
\hline $\begin{array}{l}\text { Boerhavia } \\
\text { anisophylla Torr. }\end{array}$ & G & $\mathrm{Eq}$ & Ppo & Esf & Esf & FC & $\begin{array}{l}\text { VII, } \\
19-20\end{array}$ \\
\hline $\begin{array}{l}\text { Boerhavia gracillima } \\
\text { Heimerl }\end{array}$ & G & $\mathrm{Eq}$ & Ppo & Esf & Esf & FC & $\begin{array}{c}\text { VII, } \\
21-22\end{array}$ \\
\hline $\begin{array}{l}\text { Bougainvillea glabra } \\
\text { Choisy }\end{array}$ & G & $\mathrm{Rg}, \mathrm{Bc}$ & Тср & Esf & Esf & FC & $\begin{array}{l}\text { VII, } \\
23-24\end{array}$ \\
\hline Oleaceae & & & & & & & \\
\hline $\begin{array}{l}\text { Ligustrum japonicum } \\
\text { Thunb. }\end{array}$ & M & Rt, mEq & Тср & Esf & Prol & $\mathrm{Ab}-\mathrm{Hn}$ & $\begin{array}{l}\text { VIII, } \\
1-2\end{array}$ \\
\hline \multicolumn{8}{|l|}{ Onagraceae } \\
\hline $\begin{array}{l}\text { Oenothera gracilis } \\
\text { Schrad. ex. Fisch. } \\
\text { \&C.A. Mey. }\end{array}$ & MG & $\mathrm{mGm}$ & Tpo & Obl & Obl & $\begin{array}{l}\text { Área Inap } \\
\text { Hn }\end{array}$ & $\begin{array}{l}\text { VIII, } \\
5-6\end{array}$ \\
\hline $\begin{array}{l}\text { Oenothera serrulata } \\
\text { Nutt. }\end{array}$ & MG & $\mathrm{Gr}$ & Тро & Obl & Obl & $\begin{array}{l}\text { Área Inap } \\
\text { Hn }\end{array}$ & $\begin{array}{l}\text { VIII, } \\
3-4\end{array}$ \\
\hline Papaveraceae & & & & & & & \\
\hline $\begin{array}{l}\text { Argemone ochroleuca } \\
\text { Sweet }\end{array}$ & M & Rt & Тср & Esf & Prol & $\mathrm{Ab}-\mathrm{Hn}$ & $\begin{array}{l}\text { VIII, } \\
7-8\end{array}$ \\
\hline
\end{tabular}


Acta Botanica Mexicana 113: 111-134 (2015)

Apéndice. Continuación.

\begin{tabular}{|c|c|c|c|c|c|c|c|}
\hline Taxon & $\begin{array}{l}\text { Tama- } \\
\text { ño }\end{array}$ & $\begin{array}{l}\text { Ornamen- } \\
\text { tación }\end{array}$ & $\begin{array}{l}\text { Tipo de } \\
\text { apertura }\end{array}$ & $\begin{array}{c}\text { Forma } \\
\text { granos } \\
\text { hidratados } \\
\end{array}$ & $\begin{array}{l}\text { Forma } \\
\text { granos } \\
\text { secos }\end{array}$ & $\begin{array}{l}\text { Plega- } \\
\text { miento }\end{array}$ & $\begin{array}{l}\text { Lámina, } \\
\text { Núm. de } \\
\text { figura }\end{array}$ \\
\hline \multicolumn{8}{|l|}{ Platanaceae } \\
\hline Platanus orientalis L. & M & Rt & Тср & Esf & Prol & Ab-Hn & $\begin{array}{l}\text { VIII, } \\
9-10\end{array}$ \\
\hline Plumbaginaceae & & & & & & & \\
\hline $\begin{array}{l}\text { Plumbago scandens } \\
\text { L. } \\
\text { Polygonaceae }\end{array}$ & G & $\mathrm{Cl}$ & Тср & Esf & Prol & Ab-Hn & $\begin{array}{l}\text { VIII, } \\
11-14\end{array}$ \\
\hline $\begin{array}{l}\text { Antigonon leptopus } \\
\text { Hook. \& Arn. }\end{array}$ & G & $\mathrm{Pf}$ & Tcpr & Esf & Prol & Ab-Hn & $\begin{array}{l}\text { VIII, } \\
15-16\end{array}$ \\
\hline $\begin{array}{l}\text { Polygonum aviculare } \\
\text { L. }\end{array}$ & M & $\mathrm{mEq}$ & Tcpr & Prol & Prol & Ab-Hn & $\begin{array}{l}\text { VIII, } \\
17-18\end{array}$ \\
\hline
\end{tabular}

Punicaceae

$\begin{array}{llllll}\text { Punica granatum L. } & \text { P } & \text { Fs } & \text { Tcpr } & \text { Esf } & \text { Prol Ab-Hn VI, 23- }\end{array}$

Rosaceae

Pyracantha coccinea P Ps Tcpr Esf Prol Ab-Hn VIII, M. Roem.

Solanaceae

$\begin{array}{lllllll}\text { Datura stramonium } & \text { G } & \text { Rg } & \text { Tcp } & \text { Irr } & \text { Prol } & \text { Plg-Irr }\end{array}$ L.

Nicotiana glauca

M Ps Tcp

21-22

Graham

Solanum rostratum

Dunal

Talinaceae

Talinum aurantiacum $\quad \mathrm{M} \quad \mathrm{mEq} \quad \mathrm{Pcp} \quad$ Prol Polig a Ligera IX, 3-4 Engelm.

23-24

Tamaricaceae

Tamarix gallica L. $\quad$ P $\quad$ mRt $\quad$ Tcp $\quad$ Esf $\quad$ Prol Ab-Hn IX, 5-6

Zygophyllaceae

Tribulus terrestris L. M Lf Inap Esf $\quad$ Esf Algunas IX, 7-8 veces FC 